\title{
A new tissue segmentation method to calculate 3D dose in small animal radiation therapy
}

C. Noblet ${ }^{1,2^{*}}$, G. Delpon ${ }^{1,2}$, S. Supiot ${ }^{2,3}$, V. Potiron ${ }^{2,4}$, F. Paris ${ }^{2,4}$ and S. Chiavassa ${ }^{1,2}$

\begin{abstract}
Background: In pre-clinical animal experiments, radiation delivery is usually delivered with $\mathrm{kV}$ photon beams, in contrast to the MV beams used in clinical irradiation, because of the small size of the animals. At this medium energy range, however, the contribution of the photoelectric effect to absorbed dose is significant. Accurate dose calculation therefore requires a more detailed tissue definition because both density $(\rho)$ and elemental composition $\left(Z_{\text {eff }}\right)$ affect the dose distribution. Moreover, when applied to cone beam $C T$ (CBCT) acquisitions, the stoichiometric calibration of $\mathrm{HU}$ becomes inefficient as it is designed for highly collimated fan beam CT acquisitions. In this study, we propose an automatic tissue segmentation method of CBCT imaging that assigns both density $(\rho)$ and elemental composition $\left(Z_{\text {eff }}\right)$ in small animal dose calculation.

Methods: The method is based on the relationship found between CBCT number and $\rho^{*} Z_{\text {eff }}$ product computed from known materials. Monte Carlo calculations were performed to evaluate the impact of $\rho Z_{\text {eff }}$ variation on the absorbed dose in tissues. These results led to the creation of a tissue database composed of artificial tissues interpolated from tissue values published by the ICRU. The $\rho Z_{\text {eff }}$ method was validated by measuring transmitted doses through tissue substitute cylinders and a mouse with EBT3 film. Measurements were compared to the results of the Monte Carlo calculations.
\end{abstract}

Results: The study of the impact of $\rho Z_{\text {eff }}$ variation over the range of materials, from $\rho Z_{\text {eff }}=2 \mathrm{~g} \cdot \mathrm{cm}^{-3}$ (lung) to $27 \mathrm{~g} . \mathrm{cm}^{-3}$ (cortical bone) led to the creation of 125 artificial tissues. For tissue substitute cylinders, the use of $\rho Z_{\text {eff }}$ method led to maximal and average relative differences between the Monte Carlo results and the EBT3 measurements of $3.6 \%$ and $1.6 \%$. Equivalent comparison for the mouse gave maximal and average relative differences of $4.4 \%$ and $1.2 \%$, inside the $80 \%$ isodose area. Gamma analysis led to a $94.9 \%$ success rate in the $10 \%$ isodose area with $4 \%$ and $0.3 \mathrm{~mm}$ criteria in dose and distance.

Conclusions: Our new tissue segmentation method was developed for 40kVp CBCT images. Both density and elemental composition are assigned to each voxel by using a relationship between $H U$ and the product $\rho Z_{\text {eff. }}$ The method, validated by comparing measurements and calculations, enables more accurate small animal dose distribution calculated on low energy CBCT images.

Keywords: Monte Carlo simulations, Pre-clinical radiation therapy, Tissue segmentation

\footnotetext{
* Correspondence: caroline.noblet@gmail.com

${ }^{1}$ Medical Physics Department, Institut de Cancérologie de l'Ouest Centre

René Gauducheau, 44805 Saint-Herblain, France

${ }^{2}$ CRCINA, Inserm U1232, Université de Nantes, Nantes, France

Full list of author information is available at the end of the article
}

(c) The Author(s). 2018 Open Access This article is distributed under the terms of the Creative Commons Attribution 4.0 International License (http://creativecommons.org/licenses/by/4.0/), which permits unrestricted use, distribution, and reproduction in any medium, provided you give appropriate credit to the original author(s) and the source, provide a link to the Creative Commons license, and indicate if changes were made. The Creative Commons Public Domain Dedication waiver (http://creativecommons.org/publicdomain/zero/1.0/) applies to the data made available in this article, unless otherwise stated. 


\section{Background}

Over the past few years, pre-clinical radiation therapy devices dedicated to small animals have been widely developed to reliably transpose clinical techniques to small animals $[1,2]$. Photon beam energy was reduced to $100-400 \mathrm{kVp}$ to adapt beam penetration and penumbra to the size of small animals (essentially mice and rats) and to allow the use of very small beams, as narrow as $1 \mathrm{~mm}$ in diameter. However, this medium energy range leads to a higher proportion of photoelectric effect in small animals than observed in the MV energy range in human patients. Absorbed dose continues to depend significantly on mass density, but also on elemental composition, as the photoelectric cross-section depends on the $Z_{\text {eff }}^{3-4}$ (effective atomic number) [3, 4].

In this context the analytical algorithms used to estimate absorbed dose in clinical practice at MV energy range are no longer valid. Monte Carlo methods remain the best alternative for the accurate calculation of 3D absorbed dose distributions in small animals. An accurate knowledge of tissue elemental composition is necessary to achieve a dose precision level equivalent to that of clinical practice (1-3\%) at medium energy range. The tissue distribution is basically estimated from computed tomography (CT) images. Tissue equivalent materials of known densities are scanned to obtain a CT number to mass or electronic density conversion curve. In clinical practice, such a conversion curve suffices since at MV energy range the Compton effect predominates, and it essentially depends on material density. Unfortunately, density alone is insufficient to define tissues at medium energy ranges. Two tissues with the same density but different effective atomic numbers may potentially receive significantly different absorbed doses [5]. The elemental composition of materials is therefore required.

In this study, we describe an original automatic tissue segmentation method for the calculation of absorbed dose in the context of small animal radiation therapy.

\section{Methods}

$\mathrm{HU}$ calculation applied to acquisitions with a $40 \mathrm{kVp}$ uncollimated cone beam

\section{Stoichiometric method}

To automatically obtain the elemental composition of tissues from CT images, a stoichiometric calibration method [6] was originally proposed by Schneider et al. [7]. It relies on CT scanning known materials to find a relationship between $\mathrm{CT}$ number, physical density and effective atomic number by fitting the parameters of the Jackson and Hawkes equation (Eq. 1) [8, 9].

$$
\mu=\rho N_{A} \sum_{i=1}^{n}\left(\frac{w_{i}}{A_{i}}\left(K^{\mathrm{KN}} Z_{i}+K^{\mathrm{ph}} Z_{i}^{4.62}+K^{\mathrm{sca}} Z_{i}^{2.86}\right)\right)
$$

$$
\text { CTnumber }=1000 *\left(\frac{\mu}{\mu_{\text {water }}}-1\right)
$$

$\mu=$ attenuation coefficient.

$\mathrm{i}=$ chemical element.

$\mathrm{w}=$ elemental weight.

$\rho=$ mass density.

$\mathrm{N}_{\mathrm{A}}=$ Avogadro constant.

$A_{i}=$ mass number of $i$.

$\mathrm{Z}_{\mathrm{i}}=$ atomic number of $\mathrm{i}$.

$\mathrm{K}^{\mathrm{KN}}=$ Klein-Nishina coefficient.

$\mathrm{K}^{\mathrm{ph}}=$ constant characterizing the photoelectric absorption.

$\mathrm{K}^{\mathrm{sca}}=$ constant characterizing the cross section of the coherent and incoherent scattering.

In our institution, small animal images are performed using the cone-beam CT (CBCT) scan integrated in the XRAD225Cx preclinical irradiator (Precision X-Ray Inc., CT, USA) using a non-collimated beam, rather than with a CT scan using a collimated fan beam. Eleven substitute tissue materials of known densities and elemental compositions (Gammex-RMI, WI, USA) were scanned with the CBCT imager at $40 \mathrm{kVp}$ to evaluate the stoichiometric method in our geometric settings. CBCT numbers were also calculated with Eq. 1 and compared to the experimental results.

\section{$\rho Z_{\text {eff }}$ segmentation method}

At low and medium energy range, any accurate tissue segmentation method must take into account both density $(\rho)$ and elemental composition, so the relationship between the CBCT number, $\rho$ and $Z_{\text {eff }}$ must be determined. CBCT images previously acquired from the 11 substitute tissue materials were used to test different plots of a function of $\rho$ and $Z_{\text {eff }}$ versus CBCT number. The curve $\rho Z_{\text {eff versus }}$ CBCT number led to a successful monotonic relationship (Fig. 1).

Using the set of substitute tissue materials from the Gammex-RMI phantom, a third degree polynomial equation fitted the relationship of $\rho Z_{\text {eff }}$ versus CBCT number very well, with a 0.999 correlation coefficient. Based on this $\left(\rho Z_{\text {eff }}, H U\right)$ relationship, a CBCT number can be calculated for every material of known $\rho Z_{\text {eff }}$, and reciprocally.

The proposed $\rho Z_{\text {eff }}$ assignation can be applied as follows:

i) a $40 \mathrm{kVp}$ CBCT scan of materials with known $\rho Z_{\text {eff }}$ is performed;

ii) the $\left(\mathrm{HU}, \rho \mathrm{Z}_{\mathrm{eff}}\right)$ polynomial relationship is determined;

iii) the $\mathrm{HU}$ for a list of tissues of known $\rho \mathrm{Z}_{\mathrm{eff}}$ are calculated;

iv) tissues are assigned to CBCT images based on the list in iii). 


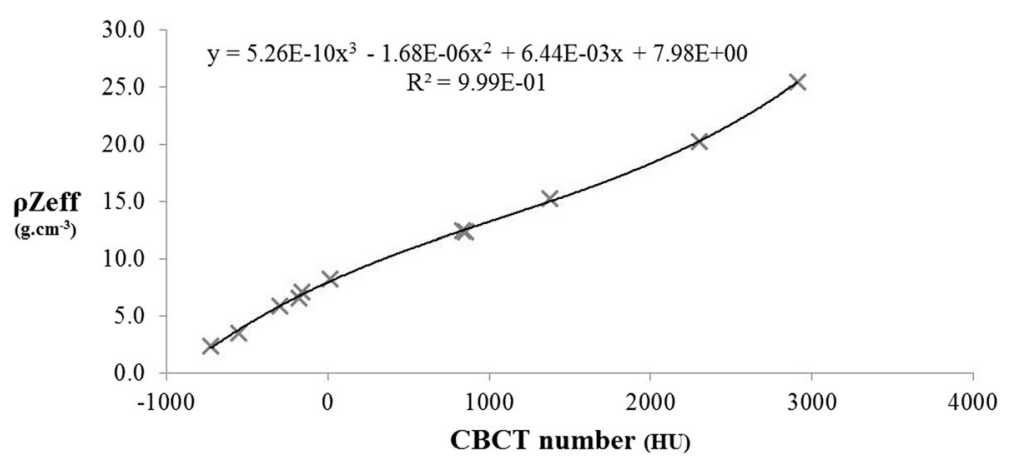

Fig. 1 CBCT number variation with the product of physical density $\rho$ and effective atomic number $Z_{\text {eff. }}$ Each $x$ represents a different insert from the Gammex-RMI phantom. The solid line represents the third degree polynomial fitting curve

\section{"Dose-equivalent" tissue calculation}

To obtain the expected dose precision level (1-3\%) with

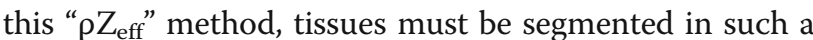
way that the dose difference between two neighboring tissues (in terms of $\rho Z_{\text {eff }}$ ) is lower than $2-3 \%$. In other words, we need a list of tissues generated with an adjusted $\rho Z_{\text {eff }}$ step.

Absorbed dose was calculated in different tissues of known $\rho Z_{\text {eff }}$ with a validated GATEv7 Monte Carlo model $[10,11]$ as follows: tissues from ICRU report 44 [12] and 46 [13] (Table 1) were attributed to a piece of $5 \times 5 \mathrm{~cm}^{2}$ and $0.5 \mathrm{~cm}$ thick tissue inserted at $1.5 \mathrm{~cm}$ depth in a $5 \times 5 \times 5 \mathrm{~cm}^{3}$ water tank. A $5 \mathrm{~mm}$ diameter circular beam of $225 \mathrm{kVp}$ was simulated. Absorbed dose in tissue was normalized to absorbed dose at the same position in a homogeneous water tank. These calculated absorbed doses were used to estimate the maximum $\rho \mathrm{Z}_{\text {eff }}$ difference between tissues required to reach a $2 \%$ dose precision. A list of tissues with a $\rho Z_{\text {eff }}$ step corresponding to this maximum difference was generated. The HU was calculated for each tissue using the $\left(\mathrm{HU}, \rho \mathrm{Z}_{\mathrm{eff}}\right)$ polynomial relationship.

\section{Validation of the tissue assignation method Transmitted dose through known materials}

In each voxel, the tissue assignment is automatically performed using the previously obtained tissue database. For each of these tissues, an HU interval has been calculated with the ( $\left.\rho \mathrm{Z}_{\text {eff }}, \mathrm{HU}\right)$ polynomial relationship illustrated in Fig. 1. However, HU noise in acquired images (up to $30 \mathrm{HU}$ ) and polynomial relationship can introduce bias, and lead to incorrect tissue assignment. In order to estimate the accuracy of our segmentation method, the dose transmitted through tissue-equivalent cylinders (Gammex-RMI, WI, USA) was measured. Indeed, the measurement of absorbed dose within a medium cannot be performed as detectors are usually calibrated in terms of dose to water and thus provide absorbed dose to water in medium. Consequently, the validation was based on transmitted doses measured with EBT3 Gafchromic films (International Specialty Products, NJ, USA). EBT3 films were positioned under the tissue-equivalent cylinders at $33 \mathrm{~cm}$ from the source (Fig. 2). The same irradiation procedure was then simulated with our MC model with two different settings:

i. MC simulations were performed by assigning the manufacturer's published compositions for the cylinders.

ii. Artificial tissues were assigned to each voxel based on our automatic segmentation method and a CBCT number obtained for each cylinder at $40 \mathrm{kVp}$. The high density cylinders exhibited a significant beam hardening effect in the CBCT scan, so it was decided to assign materials according to the CBCT number obtained at the periphery of the cylinder. This effect is very limited in preclinical radiation therapy with small animals, as bone size is smaller than a few $\mathrm{mm}$.

\section{Transmitted dose through a mouse}

An EBT3 film was positioned under the mouse to be irradiated with an anterior $2 \mathrm{~cm}$ diameter beam at $225 \mathrm{kVp}$. A CBCT scan of the mouse was performed with the EBT3 in place to ensure that the irradiation was identical with our MC model, and avoid displacement of the mouse between scanning and irradiation, which could skew the MC/EBT3 result concordance. The location of the film in the CBCT scan could then also be used to derive the absorbed dose at the same position. Four $2 \mathrm{~mm}$ diameter radio-opaque balls were positioned near the edge of the film in order to facilitate registration between measurement and simulation.

In order to subtract the dose contribution due to the CBCT scan, the procedure was repeated with a second film positioned under the mouse and then scanned at $40 \mathrm{kVp}$ but not irradiated at $225 \mathrm{kVp}$. The same calibration procedure to measure $2 \mathrm{D}$ absorbed dose with radiochromic film was applied to both films [10]. The EBT3 films were 
Table 1 Tissues from ICRU report 44 [12] and 46 [13]

\begin{tabular}{|c|c|c|c|}
\hline ICRU tissues & $\begin{array}{l}\text { Physical density } \\
\rho\left(\mathrm{g} . \mathrm{cm}^{3}\right)\end{array}$ & $Z_{\text {eff }}$ & $\rho Z_{\text {eff }}$ \\
\hline Lung inflated & 0.26 & 7.88 & 2.05 \\
\hline Adipose & 0.95 & 6.67 & 6.34 \\
\hline Yellow marrow & 0.98 & 6.56 & 6.43 \\
\hline Average soft tissue adult female & 1.02 & 7.44 & 7.59 \\
\hline Red marrow & 1.03 & 7.44 & 7.66 \\
\hline Water & 1.00 & 7.73 & 7.73 \\
\hline Average soft tissue adult male & 1.03 & 7.60 & 7.83 \\
\hline Gl tract & 1.03 & 7.71 & 7.94 \\
\hline Pancreas & 1.04 & 7.70 & 8.01 \\
\hline Breast & 1.02 & 7.88 & 8.04 \\
\hline Eyes lens & 1.07 & 7.54 & 8.07 \\
\hline Lymph & 1.03 & 7.84 & 8.07 \\
\hline Testis & 1.04 & 7.82 & 8.13 \\
\hline Brain & 1.04 & 7.88 & 8.19 \\
\hline Urinary bladder filled & 1.03 & 7.98 & 8.22 \\
\hline Kidney & 1.05 & 7.84 & 8.23 \\
\hline Ovary & 1.05 & 7.84 & 8.24 \\
\hline Muscle & 1.05 & 7.85 & 8.24 \\
\hline Lung deflated & 1.05 & 7.88 & 8.27 \\
\hline Skin & 1.09 & 7.63 & 8.31 \\
\hline Liver & 1.06 & 7.87 & 8.34 \\
\hline Spleen & 1.06 & 7.87 & 8.34 \\
\hline Heart & 1.06 & 7.95 & 8.43 \\
\hline Blood & 1.06 & 7.97 & 8.45 \\
\hline Cartillage & 1.10 & 8.33 & 9.16 \\
\hline Thyroid & 1.05 & 9.19 & 9.65 \\
\hline Spongiosa & 1.18 & 10.74 & 12.68 \\
\hline Sacrum & 1.29 & 11.46 & 14.79 \\
\hline Femur & 1.33 & 12.09 & 16.08 \\
\hline Humerus & 1.46 & 12.61 & 18.41 \\
\hline Cranium & 1.61 & 13.13 & 21.14 \\
\hline Mandible & 1.68 & 13.33 & 22.40 \\
\hline Cortical bone 5 year Child & 1.75 & 13.56 & 23.72 \\
\hline Cortical bone & 1.92 & 13.98 & 26.84 \\
\hline
\end{tabular}

calibrated at $225 \mathrm{kVp}$ in $2 \mathrm{~cm}$ deep water at the isocenter with a $10 \mathrm{~cm}$ square beam. The reference dose was calculated at the same position according to the TRS-398 dosimetric protocol [14-16] published by the International Atomic Energy Agency. Films were digitized 24 h after their irradiation with a V700 Epson scanner (Epson America Inc., CA, USA) at 200dpi resolution using three 16-bit monochrome channels to yield a Tiff image. Optical density was converted into grayscale values based on the triple channel analysis method $[17,18]$.

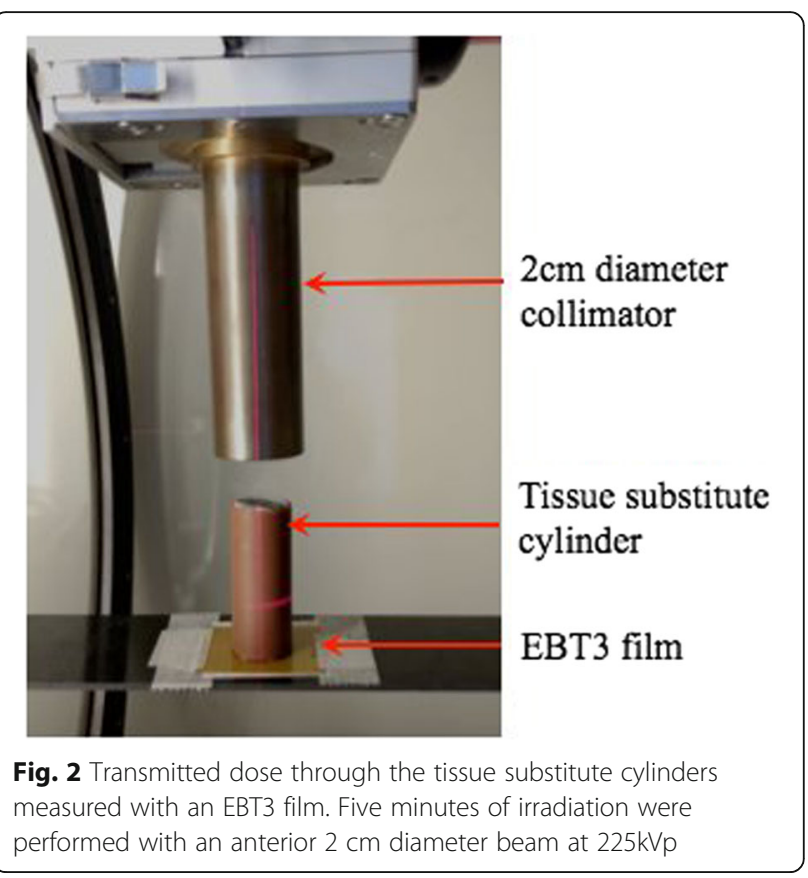

In the MC simulation, the EBT3's properties were considered to be equivalent to those of water, as they were calibrated according to absorbed dose in water. The $\rho Z_{\text {eff }}$ method was applied at each voxel representing the mouse. Simulation output resolution was set to $0.2 \mathrm{~mm} \times$ $0.2 \mathrm{~mm} \times 0.2 \mathrm{~mm}$, a trade-off between acceptable resolution and calculation time.

To compare the MC result with EBT3 measurements, a gamma analysis [19] was performed with RIT113 software (Radiological Image Technology Inc., CO, USA). The EBT3 measurement was set as the reference image and the $M C$ result as the target image. Both were normalized to the same value. We evaluated the uncertainties and dimensions for acceptable gamma criteria in a previous study [10]. The dose difference (DD) criterion was set to $4 \%$ given that the measurement uncertainty was $3.2 \%$ and the statistical uncertainty of the MC simulations was less than $1.5 \%$. Distance to agreement (DTA) was adapted from human to mouse according to image resolution (from $2 \mathrm{x} 2 \mathrm{x} 2 \mathrm{~mm}^{3}$ to $0.2 \times 0.2 \times 0.2 \mathrm{~mm}^{3}$ ) and beam size $(\mathrm{cm}$ to $\mathrm{mm})$. It was set to $0.3 \mathrm{~mm}$.

\section{Results}

$\mathrm{HU}$ calculation applied to acquisitions with a $40 \mathrm{kVp}$ uncollimated cone beam

The stoichiometric method obtained differences greater than $100 \mathrm{HU}$ and up to $480 \mathrm{HU}$ between calculated and experimental CBCT numbers, especially for materials assimilated to bony tissues (Fig. 3). 


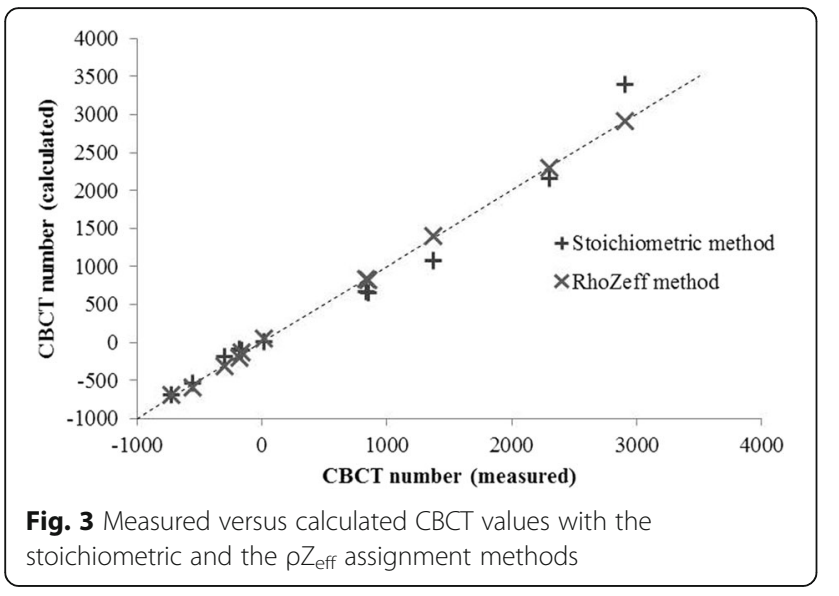

Using the polynomial fitting equation of Fig. 1, measured and calculated CBCT numbers were in good agreement for all materials, with differences of less than $40 \mathrm{HU}$ (Fig. 3).

\section{"Dose-equivalent" tissue calculation}

Figure 4 shows the absorbed dose in ICRU tissues versus $\rho Z_{\text {eff. The }} \rho Z_{\text {eff }}$ difference between tissues must be on average no larger than $0.2 \mathrm{~g} . \mathrm{cm}^{-3}$ to obtain a less than $2 \%$ dose difference. Unfortunately, the $\rho Z_{\text {eff }}$ difference is larger than $0.2 \mathrm{~g}_{\mathrm{cm}} \mathrm{cm}^{-3}$ for many tissues defined in ICRU

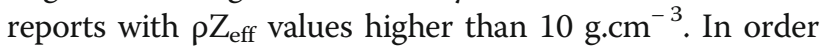
to limit the dose difference to a maximum of $2 \%$ between two neighboring tissues, 125 materials were linearly interpolated based on the $\rho Z_{\text {eff }}$ values of ICRU tissues.

The linear interpolation performed for $\rho Z_{\text {eff }}$ values varied from 2 (ICRU inflated lung) up to 27 (ICRU cortical bone) in steps of 0.2 . The elemental composition of each artificial tissue corresponded to a linear combination of the two nearest ICRU tissue neighbors.
For each of the 125 artificially created tissues for a specific $\rho Z_{\text {eff }}$ (between 2 and 27 in steps of 0.2 ):

i). The $Z_{\text {eff }}$ of the tissue is linearly interpolated based on the known $\rho Z_{\text {eff }}$ values of the nearest ICRU tissues.

ii). The mass fraction $w_{i}$ of each element $i$ is a linear interpolation of elemental mass fractions of the two nearest existing ICRU tissues (neighbors in term of $\rho Z_{\text {eff }}$ ).

iii). The effective atomic number $Z_{\text {eff }}$ is recalculated based on the interpolated $\mathrm{w}_{\mathrm{i}}$ of each element.

iv). If the recalculated $Z_{\text {eff }}$ differed by more than $5 \%$ from the expected $Z_{\text {eff }}$ value in (i), the selected weight of element $\mathrm{i} \mathrm{w}_{\mathrm{i}}$ was iteratively increased or decreased by a 0.001 step depending on whether the difference was either positive or negative:

o for $\rho Z_{\text {eff }}<10$ : carbon and oxygen.

o for $\rho Z_{\text {eff }}>10$ : calcium and phosphor.

v). The mass fraction sum of all elements was recalculated. This must be equal to 1 , or the hydrogen mass fraction is modified, given that hydrogen's atomic number $(Z=1)$ has a negligible impact on $Z_{\text {eff }}$ value.

vi). $\rho$ is deducted from the expected value of $\rho Z_{\text {eff }}$ and the calculated value of $Z_{\text {eff. }}$.

There is no anatomical meaning in the definition of these materials. These artificial materials should be considered as "dose-equivalent" tissues with properties that lead to the same absorbed dose, rather than actual tissues of unknown composition. A corresponding CT number range was calculated with the $\left(\rho Z_{\text {eff }}, H U\right)$ relationship for each of these 125 artificial tissues.

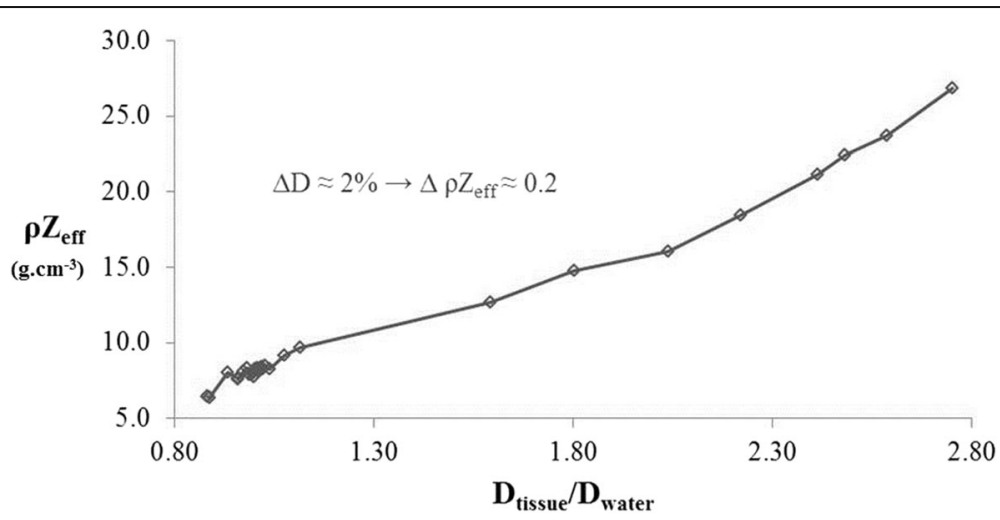

Fig. $4 \rho Z_{\text {eff }}$ variation with absorbed dose in ICRU tissues (see Table 1). MC dose calculations were performed for an anterior $5 \mathrm{~mm} 225 \mathrm{kVp}$ beam in a $5 \times 5 \times 5 \mathrm{~cm}^{3}$ water tank with a $5 \mathrm{~mm}$ thick tissue insert at $1.5 \mathrm{~cm}$ depth in water. Absorbed dose in tissue was normalized to absorbed dose at the same position in the homogeneous water tank. This plot shows that a $0.2 \rho Z_{\text {eff }}$ interval between two neighboring tissues is required to reach approximately $2 \%$ dose calculation precision 
In summary, the reference ICRU tissues were only used to determine the maximum $\rho Z_{\text {eff }}$ difference needed between two neighboring tissues in order to obtain an absorbed dose precision in animals of less than $2 \%$ (Fig. 4), and to interpolate 125 artificial tissues with $\rho Z_{\text {eff }}$ values from 2 g.cm ${ }^{-3}$ up to 27 g.cm ${ }^{-3}$ in steps of 0.2 , as determined by these obtained $\rho Z_{\text {eff }}$ differences.

\section{Validation of the tissue assignation method Transmitted dose through known materials}

The use of the manufacturer's data in the MC computations yielded good agreement between the MC results and EBT3 transmission measurements (Table 2). The maximum relative difference was found to be $3 \%$ in all materials. The mean relative difference of exit absorbed doses was less than $1.5 \%$. Those results confirmed the ability of our MC model and our film dosimetry method to calculate absorbed doses with good accuracy whatever the material. Our assignment method based on the $\left(\mathrm{HU}, \rho \mathrm{Z}_{\text {eff }}\right)$ relationship, with no a priori knowledge of the material, then found transmitted absorbed doses to be in good agreement with EBT3 measurements. Maximum difference was 3.6\%. The mean relative difference of exit absorbed doses was about $1.6 \%$ (Table 2).

\section{Transmitted dose through a mouse}

Measured absorbed dose in the EBT3 film was compared to the same coronal plane extracted from the MC 3D dose distribution (Fig. 5). EBT3 measurements and $\mathrm{MC}$ results agreed very well, with dose differences less than 0.3Gy, mainly in the field periphery (Fig. 6). Inside the $80 \%$ isodose area (the area where absorbed dose is more than $80 \%$ of the maximum dose) a $4.4 \%$ maximum and a $1.2 \%$ mean relative differences were found, corresponding to a 0.04Gy maximum dose difference. Gamma analysis revealed a $94.9 \%$ success rate in the $10 \%$ isodose area with $4 \%$ DD and $0.3 \mathrm{~mm}$
DTA criteria. Failed pixels were mainly localized in the penumbra where dose gradient was high. However, a profile line along a diameter (Fig. 6d) showed these discrepancies were slight.

\section{Discussion}

In small animal radiotherapy practice, a limited number of tissues are generally assigned, such as air, lung, muscle and cortical bone, using differences in CT numbers. However, many studies have showed that this method leads to tissue mis-assignment and potentially large dosimetric errors. Chow et al. [3] calculated up to $400 \%$ deviation at $225 \mathrm{kVp}$ between absorbed dose calculated in a homogeneous mouse and in a mouse in which bone was considered. Verhaegen et al. [20] highlighted that an incorrect tissue assignment could lead to dose error greater than $40 \%$ at $250 \mathrm{kVp}$ whereas less than $10 \%$ error was found at $6 \mathrm{MV}$ and $15 \mathrm{MV}$. Zhou et al. [21] have demonstrated that 47 different bony tissues were needed with a $120 \mathrm{kVp}$ beam to reach $2 \%$ dose accuracy. Bazalova et al. [4] suggest that 92 tissues must be defined to obtain $2 \%$ accuracy for dose calculation at 225 $\mathrm{kVp}$, showing that a simplistic 4-8 tissue assignment could lead to differences of more than $30 \%$ when compared to a dose distribution calculated with 39 tissues. Those previous studies showed a large number of materials must be defined to reach a satisfactory dose accuracy in the range of $1-3 \%$.

Our tissue segmentation method principle essentially follows the stoichiometric calibration method, fully described by Vanderstraeten et al. [6]. This method has particularly found application in proton therapy, where material attribution is also a critical parameter [22, 23], but suffers significant error in $\mathrm{HU}$ calculations based on CBCT acquisitions. The stoichiometric calibration was originally designed for a highly collimated fan beam [8], but it fails when applied to CBCT acquisitions whose divergent broad beam produces more scattered radiation. No gold standard

Table 2 Transmitted dose through tissue substitute materials, measured and simulated with manufacturer's data (Gammex) and the $\rho Z_{\text {eff }}$ tissue assignment method. EBT3 measurement uncertainty was 3.2\% [10]

\begin{tabular}{|c|c|c|c|c|c|c|c|c|c|}
\hline \multirow[b]{2}{*}{$\begin{array}{l}\text { Tissue } \\
\text { substitutes }\end{array}$} & \multirow[b]{2}{*}{ EBT3 (Gy) } & \multicolumn{4}{|c|}{ Manufacturer's data } & \multicolumn{4}{|c|}{$\rho$ Zeff based tissue assignation method } \\
\hline & & $\begin{array}{l}\text { MC } \\
\text { dose (Gy) }\end{array}$ & $\begin{array}{l}\text { Stat. } \\
\text { Uncert. }\end{array}$ & $\begin{array}{l}\text { MC/EBT3 relative } \\
\text { difference }\end{array}$ & $\begin{array}{l}\text { MC/EBT3 absolute } \\
\text { difference (Gy) }\end{array}$ & $\begin{array}{l}\text { MC } \\
\text { Dose (Gy) }\end{array}$ & $\begin{array}{l}\text { Stat. } \\
\text { Uncert. }\end{array}$ & $\begin{array}{l}\text { MC/EBT3 relative } \\
\text { difference }\end{array}$ & $\begin{array}{l}\text { MC/EBT3 absolute } \\
\text { difference (Gy) }\end{array}$ \\
\hline CT solid water & 4.58 & 4.61 & $1.4 \%$ & $0.5 \%$ & 0.03 & 4.51 & $1.3 \%$ & $-1.5 \%$ & -0.07 \\
\hline Inner bone & 3.44 & 3.50 & $1.5 \%$ & $1.7 \%$ & 0.06 & 3.41 & $1.5 \%$ & $-1.0 \%$ & -0.03 \\
\hline Cortical bone & 1.40 & 1.38 & $2.0 \%$ & $-1.8 \%$ & -0.02 & 1.42 & $2.1 \%$ & $1.3 \%$ & 0.02 \\
\hline CB2 50\% & 1.99 & 1.93 & $1.8 \%$ & $-3.0 \%$ & -0.06 & 2.02 & $1.8 \%$ & $1.5 \%$ & 0.03 \\
\hline CB2 30\% & 2.73 & 2.72 & $1.6 \%$ & $-0.4 \%$ & -0.01 & 2.80 & $1.6 \%$ & $2.6 \%$ & 0.07 \\
\hline Breast & 4.93 & 4.91 & $1.3 \%$ & $-0.4 \%$ & -0.02 & 4.75 & $1.3 \%$ & $-3.6 \%$ & -0.18 \\
\hline B200-bone & 3.41 & 3.49 & $1.5 \%$ & $2.4 \%$ & 0.08 & 3.45 & $1.5 \%$ & $1.1 \%$ & 0.04 \\
\hline Adipose & 5.29 & 5.35 & $1.3 \%$ & $1.3 \%$ & 0.07 & 5.29 & $1.3 \%$ & $0.1 \%$ & 0.01 \\
\hline
\end{tabular}




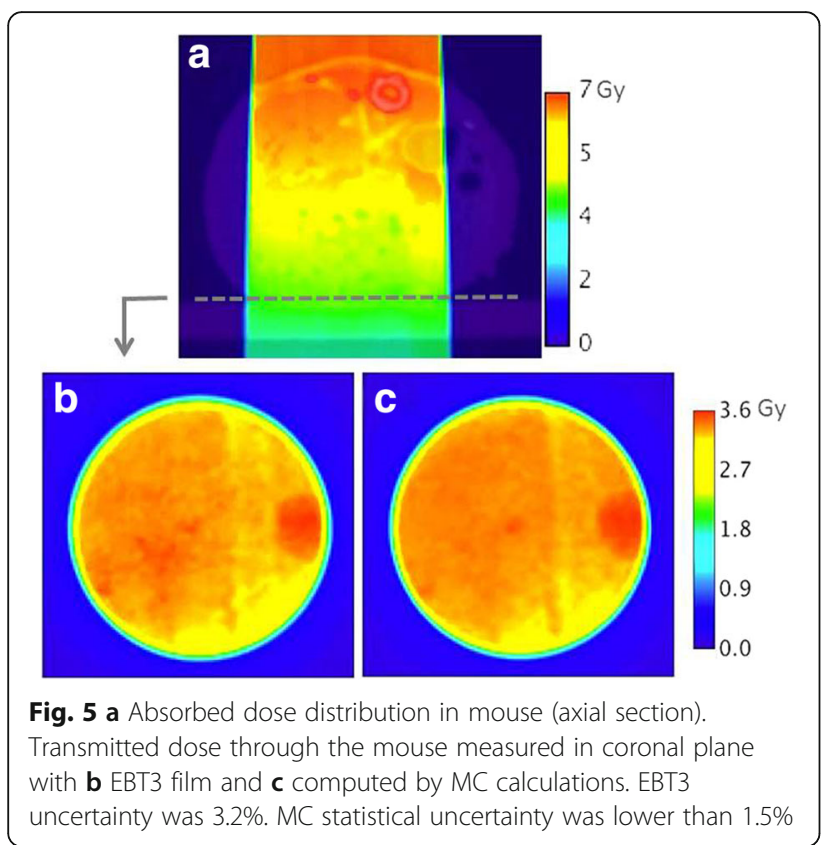

tissue segmentation method exists for $\mathrm{CBCT}$ images and various approaches have been explored, such as, recently, dual energy CBCT [24]. Our method, based on the (HU, $\left.\rho Z_{\text {eff }}\right)$ relationship, showed satisfactory results for dose calculation at $225 \mathrm{kV}$ based on $40 \mathrm{kVp}$ CBCT images. It was validated by measuring transmitted doses through known materials and unknown materials using respectively tissue-equivalent cylinders and a real mouse.
Elemental composition and densities for small animal tissues are still unknown. In the absence of data on small animal tissue composition, the use of human tissue to assign values for small animals is inevitable but remains questionable. However, the use of indirect segmentation methods, such as the stoichiometric or $\rho Z_{\text {eff }}$ methods allows the concept of "dose-equivalent" tissues to be defined. These assigned tissues are not anatomically consistent: they are artificially generated with the aim of being sufficiently discriminating between small animal tissues to provide accurate dose calculation.

\section{Conclusion}

The major dosimetric impact of heterogeneities in small animal tissues means that tissue assignment is a primordial parameter for attaining reliable dose distributions for absorbed dose calculation in pre-clinical practice. We have shown that an automatic voxel by voxel tissue assignment method based on a third degree polynomial relationship between $\mathrm{CT}$ numbers $(\mathrm{HU})$ and $\rho \mathrm{Z}_{\text {eff }}$ is effective at reducing the dose distribution errors probable with simpler tissue assignment methods. Dose differences of less than $4 \%$ were found between measured and calculated dose transmitted though several tissue substitute materials with this new tissue attribution method. Less than $4.4 \%$ dose difference was obtained inside the $80 \%$ isodose area between measured and simulated dose transmitted through a mouse, suggesting satisfactory tissue assignment.

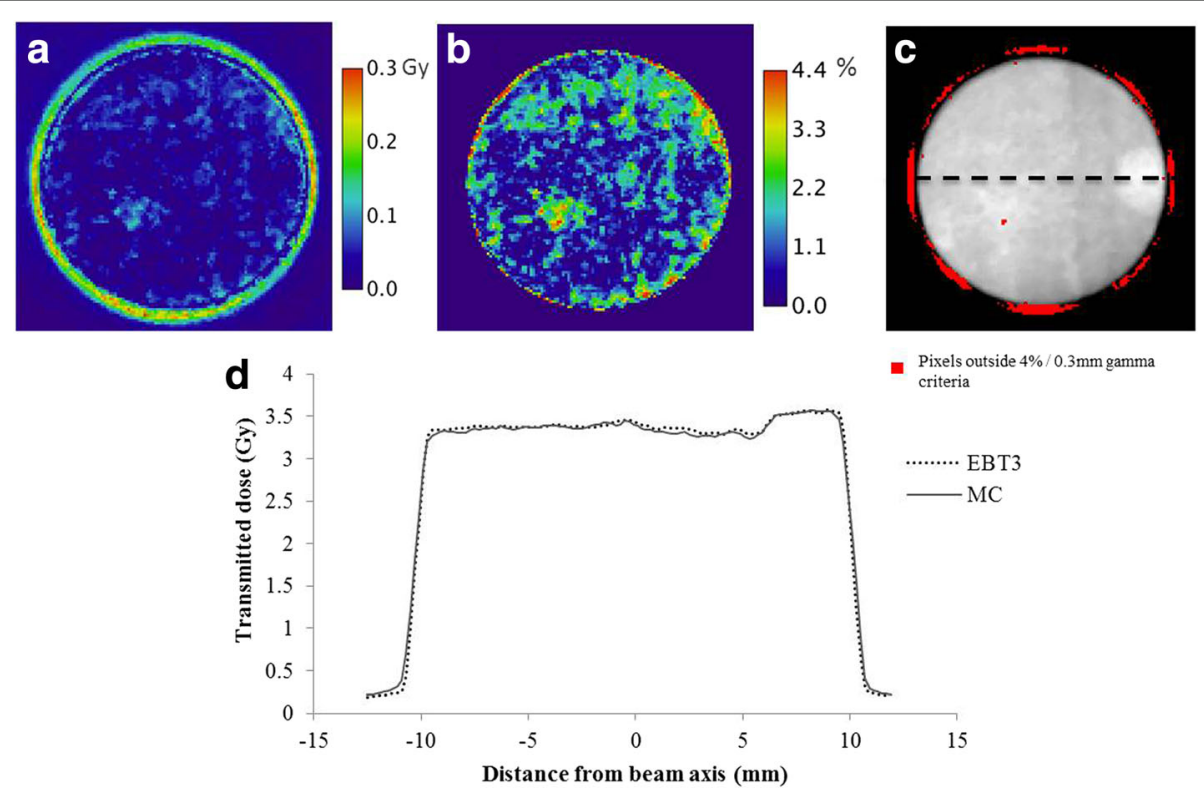

Fig. 6 a Absorbed dose difference (Gy) between EBT3 and MC results. b Relative dose difference (\%) between EBT3 and MC results performed inside the $80 \%$ isodose area. c Gamma analysis performed with 4\% DD and $0.3 \mathrm{~mm}$ DTA, revealing a success rate of $94.9 \%$. d Measured and calculated horizontal dose profiles along a diameter plotted with the dashed line on (c) 


\section{Abbreviations}

CBCT: Cone Beam Computed Tomography; CT: Computed Tomography; HU: Hounsfield unit; MC: Monte Carlo; $Z_{\text {eff. }}$ effective atomic number; $\rho$ : mass density

\section{Acknowledgements}

Not applicable

\section{Funding}

Financial support was partly provided by a Nantes Metropole grant and "Physics, Mathematics and Engineering sciences applied to the Cancer Research" grant (MC SMART project n A12149NS).

\section{Availability of data and materials}

Data sharing is not applicable to this review article as no datasets were generated or analysed during the current study.

\section{Authors' contributions}

CN carried out literature research, performed MC simulations and XRAD irradiations, participated in the tissue assignment method implementation, MC and measurement analysis and interpretation of the results, generated tables and figures, drafted the manuscript. GD participated in the tissue assignment method implementation, in interpretation of the results, design of the figures, literature research and drafted the manuscript. SS FP and VP set out the biological objectives of the study in terms of dosimetry, participated in interpretation of the results and drafted the manuscript. VP participated in XRAD irradiations. SC drafted the manuscript, performed MC simulations and XRAD irradiations, participated in the tissue assignmen method implementation, MC and measurement analysis and interpretation of the results, design of the figures, literature research. All contributors have read and approved the final manuscript.

\section{Ethics approval and consent to participate}

Not applicable

\section{Consent for publication}

Not applicable.

\section{Competing interests}

The authors declare that they have no competing interests.

\section{Publisher's Note}

Springer Nature remains neutral with regard to jurisdictional claims in published maps and institutional affiliations.

\section{Author details}

'Medical Physics Department, Institut de Cancérologie de l'Ouest Centre René Gauducheau, 44805 Saint-Herblain, France. ${ }^{2}$ CRCINA, Inserm U1232, Université de Nantes, Nantes, France. ${ }^{3}$ Radiotherapy Department, Institut de Cancérologie de l'Ouest Centre René Gauducheau, 44805 Saint-Herblain, France. ${ }^{4} \mathrm{LABCT}$, Institut de Cancérologie de I'Ouest Centre René Gauducheau, 44805 Saint-Herblain, France.

Received: 20 September 2017 Accepted: 11 February 2018 Published online: 26 February 2018

\section{References}

1. Verhaegen F, Granton P, Tryggestad E. Small animal radiotherapy research platforms. Phys Med Biol. 2011;56:R55-83.

2. Koontz BF, Verhaegen F, De Ruysscher D. Tumour and normal tissue radiobiology in mouse models: how close are mice to mini-humans? Br J Radiol. 2016;26:20160441.

3. Chow JCL, Leung MKK, Lindsay PE, Jaffray DA. Dosimetric variation due to photon beam energy in the small-animal irradiation: a Monte Carlo study. Med Phys. 2010;37:5322-9.

4. Bazalova M, Graves EE. The importance of tissue segmentation for dose calculations for kilovoltage radiation therapy. Med Phys. 2011:38:3039-49.

5. Noblet C, Chiavassa S, Paris F, Suhard J, Lisbona A, Delpon G. Impact of tissue assignment for preclinical radiotherapy: a dose-volume histogram analysis. Radiother Oncol. 2014;111:S68.
6. Vanderstraeten B, Chin PW, Fix M, Leal A, Mora G, Reynaert N, Seco J, Soukup M, Spezi E, De Neve W, Thierens H. Conversion of CT numbers into tissue parameters for monte carlo dose calculations: a multi-centre study. Phys Med Biol. 2007:52:539-62.

7. Schneider U, Pedroni E, Lomax A. The calibration of CT Hounsfield units for radiotherapy treatment planning. Phys Med Biol. 1996:41:111-24.

8. Jackson DF, Hawkes DJ. X-ray attenuation coefficients of elements and mixtures. Phys Rep. 1981;70(3):169-233.

9. Yohannes I, Kolditz D, Langner O, Kalender WA. A formulation of tissue- and water-equivalent materials using the stoichiometric analysis method for CTnumber calibration in radiotherapy treatment planning. Phys Med Biol. 2012;57:1173-90.

10. Noblet C, Chiavassa S, Smekens F, Sarrut D, Passal V, Suhard J, Lisbona A, Paris F, Delpon G. Validation of fast Monte Carlo dose calculation in small animal radiotherapy with EBT3 radiochromic films. Phys Med Biol. 2016:61: 3521-35.

11. Smekens F, Létang JM, Noblet C, Chiavassa S, Delpon G, Freud N, Rit S, Sarrut D. Split exponential track length estimator for Monte-Carlo simulations of small-animal radiation therapy Phys. Med Biol. 2014;59:7703-15.

12. International Commission on Radiation Units and Measurements (ICRU). Report 44. Tissue substitutes in radiation dosimetry. Washington: International Commission on Radiation Units and Measurements; 1989.

13. International Commission on Radiation Units and Measurements (ICRU). Report 46. Photon, electron, proton and neutron interaction data for body tissues. Washington: International Commission on Radiation Units and Measurements; 1992.

14. Andreo P, Burns DT, Hohlfield K, Huq MS, Kanai T, Laitano F, Smyth V, Vynckier $\mathrm{S}$. Absorbed dose determination in external beam radiotherapy, an international code of practice for dosimetry based on standards of absorbed dose to water technical report series no 398. Vienna: IAEA; 2000

15. Perichon N, Rapp B, Denoziere M, Daures J, Ostrowsky A, Bordy JM. Comparison between absorbed dose to water standards established by water calorimetry at the LNE-LNHB and by application of international airkerma based protocols for kilovoltage medium energy $x$-rays. Phys Med Biol. 2013:58:2787-806.

16. Rapp B, Perichon N, Denoziere M, Daures J. Ostrowsky a and Bordy J M. The LNE-LNHB water calorimeter for primary measurement of absorbed dose at low depth in water: application to medium-energy x-rays Phys Med Biol. 2013;58:2769-86.

17. Micke A, Lewis DF, Yu X. Multichannel film dosimetry with nonuniformity correction. Med Phys. 2011;38:2523-34.

18. van Hoof S, Granton P, Landry G, Podesta M, Verhaegen F. Evaluation of a novel triple-channel radiochromic film analysis procedure using EBT2. Phys Med Biol. 2012:57:4353-68.

19. Low DA, Harms WB, Mutic S, Purdy JA. A technique for the quantitative evaluation of dose distributions. Med Phys. 1998:25:656-61.

20. Verhaegen F, Devic S. Sensitivity study for CT image use in Monte Carlo treatment planning. Phys Med Biol. 2005:50:937-46.

21. Zhou H, Keall PJ, Graves EE. A bone composition model for monte carlo xray transport simulations. Med Phys. 2008:36:1008-18.

22. De Marzi L, Lesven C, Ferrand R, Sage J, Boulé T, Mazal A. Calibration of CT Hounsfield units for proton therapy treatment planning: use of kilovoltage and megavoltage images and comparison of parameterized methods. Phys Med Biol. 2013;58:4255-76.

23. Yang M, Zhu XR, Park PC, Titt U, Mohan R, Virshup G, Clayton JE, Dong L. Comprehensive analysis of proton range uncertainties related to patient stopping-power-ratio estimation using the stoichiometric calibration. Phys Med Biol. 2012;57:4095-115.

24. Vaniqui A, Schyns LEJR, Almeida IP, van der Heyden B, van Hoof SJ, Verhaegen $F$. The impact of dual energy CT imaging on dose calculations for pre-clinical studies. Radiat Oncol. 2017:12:181 